\title{
EVALUATION THE PERFORMANCE OF DOSE CALCULATION ALGORITHMS FOR HOMOGENEOUS AND HETEROGENEOUS PHANTOMS IN SBRT
}

\author{
KH. T. ERIEBA ${ }^{3}$, N. DEIAB ${ }^{1}$, M. MAHMOUD ${ }^{1}$, A. ELDIB ${ }^{2,3}$ AND M. SHOSHA ${ }^{1 *}$ \\ (1)Department of Radiotherapy and Nuclear Medicine, National Cancer Institute, Cairo University, Cairo-Egypt \\ (2) Fox Chase Cancer Center, Philadelphia, PA, USA (3) Department of physics, Al Azhar University, Faculty of \\ Science, Cairo-Egypt
}

\begin{abstract}
Purpose: In Stereotactic body radiation therapy (SBRT), higher dose per fraction is delivered to patients and thus the need for an accurate dose computation is further elevated. So, the aim of this study is to evaluate the performance of the algorithms implemented in our treatment planning system.

Methods: In this study different phantoms were designed to model the lung with a small tumor volume of size that are usually encountered in SBRT. Solid water and cork slabs were used in constructing the phantom body. Perspex pieces of different diameters were created to be inserted in precut holes in the cork to simulate the tumor. The various dose calculation algorithms that are implemented in our CMS XIO planning system were then used for dose calculation inside the target.

Results: A significant discrepancies between maximum, minimum and mean dose for PTV was found between FFT Convolution, Fast superposition and superposition algorithms in dose calculation for the lung tumor. The FFT Convolution algorithms have higher calculated minimum dose than that predicted by the superposition and the fast superposition algorithm. A percentage difference up to $13.4 \%$ was obtained between the FFT Convolution and the superposition algorithm for the estimated maximum dose. Superposition and fast superposition showed little discrepancies for maximum, minimum and mean dose for the PTV.

Conclusion: It is very critical to address the accuracy of dose computation for SBRT as a significant dose overestimation can occur inside the target due to the heterogeneous geometries.
\end{abstract}

Key Words: Dose calculation algorithms, Heterogeneous Phantoms, SBRT

\section{INTRODUCTION}

In radiation therapy, precision and accuracy of treatment planning and delivery are of great importance in achieving tumor eradication and in sparing healthy tissue from unnecessary radiation dose. ${ }^{1}$ The accuracy of dose computation within the lungs depends strongly on the performance of the calculation algorithm in regions of electronic disequilibrium that arise near tissue inhomogeneities with large density variations. ${ }^{2}$

A good understanding of the underlying radiation physics as it applies to a wide variety of irradiation conditions is necessary in order to ensure a dose result, which can be trusted for clinical decision-making. Unfortunately, dose algorithms are often the most hidden elements of the treatment planning software because of proprietary reasons. In recent years, the sophistication and complexity of clinical treatment planning and treatment planning systems has increased significantly, particularly including three-dimensional (3D) treatment planning systems, and the use of conformal treatment plan- ning and delivery techniques. This has led to the need for a comprehensive set of quality assurance guidelines that can be applied to clinical treatment planning. These guidelines are well discussed in literature. ${ }^{3,4}$

Experimental testing for dose distributions in water phantom and for inhomogeneity corrections will help to examine algorithms. In patient however, the beam may transverse layers of fat, bone, muscle, lung and air. The presence of these inhomogeneities will produce changes in the dose distribution, depending on the type and the amount of the material present and the quality of radiation. The algorithms used must account for the inhomogeneities present in the body. ${ }^{5}$ It should also take in account the lack of lateral electronic equilibrium in small field dosimetry as encountered with Stereotactic body radiation therapy (SBRT). Several authors have studied the influence of inhomogeneity corrections on dose distributions specifically for stereotactic treatments of lung cancer. ${ }^{6-10}$ 
Stereotactic refers to precise positioning of the target volume in three dimensional spaces. The target volume is usually localized in space using some external frame of reference, which can be related to the treatment delivery system. This makes image guided treatment delivery crucial for the success of SBRT and for keeping side effects to the lowest possible level. The term 'body' is used to distinguish the technique from treatments performed in the brain and skull base called intracranial stereotactic radiosurgery (SRS) or intracranial stereotactic radiotherapy (SRT) where the treatment accuracy can be related to positioning of the rigid skull with high reliability. ${ }^{11}$

In SBRT higher dose per fraction is delivered to patients and thus the need for an accurate dose computation is further elevated. Lung tissue is special challenge for dose calculation algorithms especially in case of stereotactic body radiation therapy (SBRT) due to small field size in combination with large variation in tissue density. ${ }^{24}$ In this study we evaluate the performance of the algorithms implemented in commercial available treatment planning systems. ${ }^{8}$

\section{MATERIAL AND METHODS}

\section{Designed phantoms}

We have designed a variety of phantoms representing homogeneous and inhomogeneous situations as a way of modeling situations usually encountered in human body; applied phantoms are schematically illustrated in Figure (1). Dose calculation were done in each of those phantoms and the accuracy of the algorithms was then determined based on measurements.

\section{Homogeneous cubic phantom:}

A homogeneous cubic phantom was designed from tissue equivalent materials to mimic the dose deposition in homogenous situations. The homogenous designed phantom was named as phantom (1). It consisting of slabs of polystyrene and the ion chamber positioned at depth $10 \mathrm{~cm}$ in the drilled solid water slab. Phantom is shown in figure (1).

\section{Heterogeneous cubic phantom:}

Three Heterogeneous phantoms were designed to model the inhomogeneous situation faced in lung tumors treatment. Each phantom consists of cork embedded in four thick solid water slabs (4 cm thickness) with a central cylindrical polystyrene insert to mimic a tumor inside the lung. It also incorporated two cylindrical polystyrene to represent organs at risk. A specially drilled solid cork slab was designed to have a cavity that accommodates our ion chamber for dose measurements. In each of the three phantoms, the location of this slab was varied to represent different points of dose measurement around our target.

Phantom (2): The drilled solid cork positioned at $4 \mathrm{~cm}$ depth. This represents a point of dose measurement distal to our target.

Phantom (3): The drilled solid cork positioned beneath the cylindrical tumor. This represents a point of dose measurement proximal to our target.

Phantom (4): The drilled solid cork positioned at the deepest location with the cork part of the phantom. This represents another distal point of dose measurement.

\section{Dose calculation algorithms}

The choice of dose calculation algorithms is an important consideration when using the different planning methodologies and comparing one method with another. ${ }^{12}$ Convolution and superposition methods involve a convolution equation that separately considers the transport of primary photons and that of the scatter photon and electron emerging from the primary photon interaction. To understand convolution algorithms we need to define the following functions; Energy fleunce, Total energy release in the media (TERMA) and scatter Kernels. Energy fleunce function is the number of crossing photons of certain energy in unit surface area. TERMA function is defined as the kinetic energy released per unit mass in the form of electrons set in motion by photons. Scatter kernels is the energy spread from a scattering point to downstream point of interest. The product of the TERMA function and the dose kernel when integrated (convolved) over a volume gives the dose. ${ }^{3,13}$ 
Three different algorithms are implemented in the CMS XIO planning system: namely Convolution algorithm, superposition algorithm and fast superposition algorithm. This can be briefly described as follows:-

\section{b1. Convolution algorithm:}

The energy deposition kernels are interpolated from spherical to Cartesian coordinates on a common grid with the TERMA to perform FFT convolution. Sampling and interpolation of kernels from spherical to Cartesian coordinates is complicated by steep kernel gradients. Adaptive quadrature techniques ensure that the correct energy at and near the interaction point is represented in the Cartesian coordinates. ${ }^{12}$

\section{b2.Superposition algorithm:}

The XiO superposition dose deposition method is an adaptation of the "collapsed cone" dose calculation method descried by Miften et al. ${ }^{14}$ The "Collapsed Cone" works as follows: all energy released into coaxial cones of solid angle $\Omega$ from volume elements on the axis is rectilinearly transported, attenuated and deposited in elements on that axis. This allows for accurate computation of the dose deposited to all voxels while performing many less computations. ${ }^{15}$

\section{b3.Fast superposition algorithm:}

It is a superposition algorithm with an added mathematical modification to speed up the dose calculation process. Spherical kernels, or "dose spread arrays", are cylindrically symmetric and defined in terms of rays traced along zenith and azimuth angles. The spherical kernel computation has been augmented with the ability to combine (select and sum) adjacent zenith rays in the kernel. Thus, it is possible to limit the number and direction of zenith rays for the purpose of optimizing speed/accuracy tradeoffs: The more the rays, the slower and more accurate the calculation: the fewer the rays, the faster and less accurate the calculation. ${ }^{12}$

In this study, we compared all of the three algorithms implemented in XIO treatment planning system. The four designed phantoms were CT imaged and targets were contoured in our planning system work station. Then SBRT treat- ment plans were generated using photon beams of $6 \mathrm{MV}$ photon beam energy. Measurements were preformed with calibrated ionization chamber to find absolute dose in a point inside

\section{Irradiation Technique}

The treatment plans generated was a box technique comprising Four-treatment fields. All treatment plans fields were narrow coplanar isocentric beams incident on the phantom from the following directions; $0^{\circ}, 90^{\circ}, 180^{\circ}$ and $270^{\circ}$ as shown in Fig. (2).

The fields were initially shaped to the tumor volume without adding a Planning Target Volume (PTV) margin, as we assumed no movement of the phantom and target. We have neglected uncertainties in tumor position caused by breathing and instead assumed that the tumor is in fixed position relative to the beams; this situation corresponds approximately to the use of gating or breath-hold techniques. The issue of variable tumor position and deforming anatomy has been already covered by other investigators. ${ }^{16-18}$

Experimental Measurements were done for all of our SBRT plans. This step was done to meet our aim of assessing the accuracy of the three algorithms in different situations. All measured doses were tabulated and compared to the calculated dose and the deviation of our calculations from measurement was determined for all algorithms.

\section{RESULTS}

Xio planning system performed the dose calculations utilizing the three algorithms for all of the SBRT plans. Ion measurement was done to get the dose at specified points which are then compared to that calculated in Xio system. Ionization chamber measurements in the different phantoms were compared to that calculated by the different algorithms.

Tables 1 summarizes the percentage point dose deviation between measured and calculated dose for the SPRT plans, Convolution algorithms showed better results in phantom 1 (homogeneous Phantom), Fast superposition show the minimum deviation for phantom 2, 3 and 4 (Heterogeneous phantom), Superposition showed good results for all phantoms and SBRT plans. 
A significant discrepancy between maximum, minimum and mean for target was found between three algorithms in dose calculation for virtual lung tumor. Figure 4 summarizes our results regarding the comparison between maximum, minimum and mean dose for PTV predicted by the different algorithms. The deviation in minimum dose can reach up to $56.4 \%$ with FFT Convolution algorithms compared to superposition algorithms. The deviation in mean dose to the target is up $21.9 \%$ with FFT Convolu- tion compared to superposition algorithms. The maximum dose to the target shows overestimation for convolution algorithm up to $13.4 \%$ and $15.2 \%$ for superposition and fast superposition respectively. Superposition and fast superposition showed little discrepancies for maximum, minimum and mean dose for target.

Superposition and fast superposition are showing little discrepancies for maximum, minimum and mean dose for target.

Table 1: Percentage point dose variation between measured and calculated.

\begin{tabular}{||c|c|c|c|c|}
\hline \multirow{2}{*}{ Algorithms } & \multicolumn{4}{|c||}{ Phantoms } \\
\cline { 2 - 5 } & 1 & 2 & 3 & 4 \\
\hline Sup. & 3.36 & -5.2 & -5.78 & -3.76 \\
\hline Fast. & 4.19 & -6.2 & -5.78 & -4.42 \\
\hline Con. & 4.6 & -8.5 & -12.5 & -13.1 \\
\hline \hline
\end{tabular}

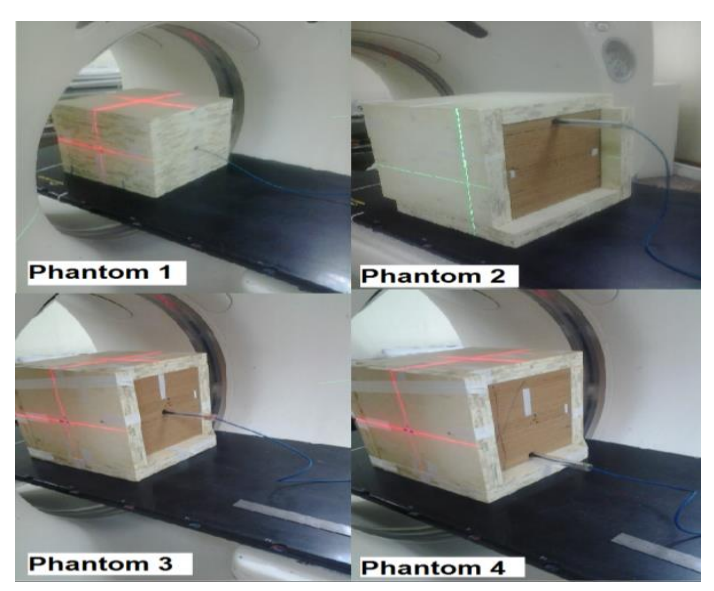

Figure (1) Diagram illustrated a variety of phantoms.

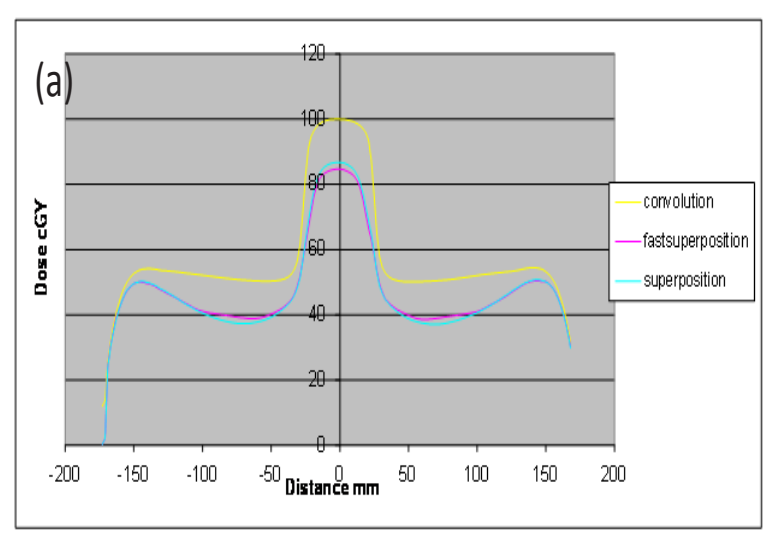

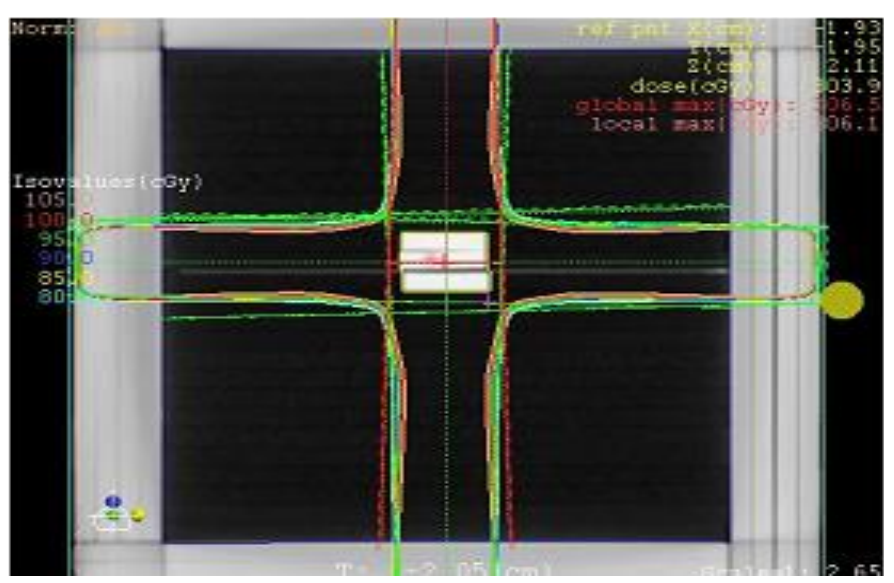

Fig (2): The layout of the virtual phantom and an example of a dose distribution from a central slice in the phantom.

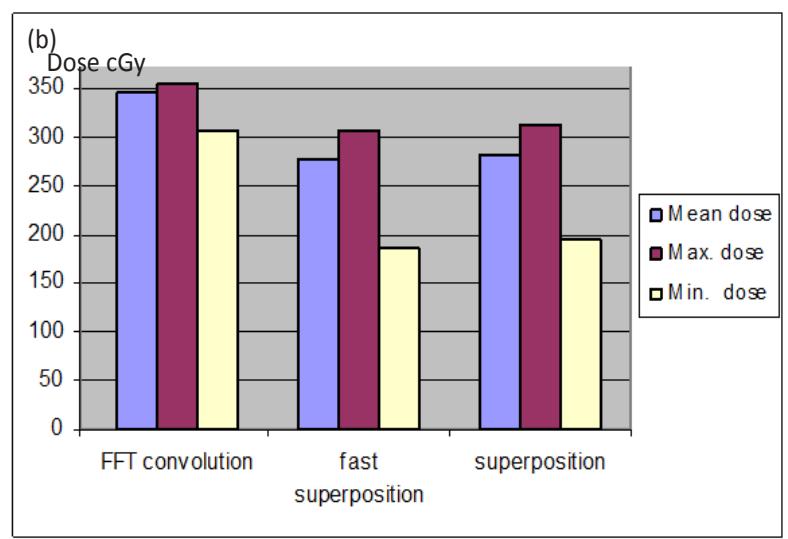

Fig (3): A comparison of the SBRT plans calculated by the different algorithms (plans were normalized to maximum dose). a) Profile taken across the target, b) Histogram plotted for the calculated maximum, minimum, and mean dose from the different algorithms. 


\section{DISCUSSION}

This study explored the discrepancies in calculated SBRT target dose that could occur when using different algorithms. It is well known that small field dosimetry is challenging due to the lack of lateral electronic dose equilibrium. This is due to the very small size of the field compared to the range of the scattered radiation. Treatment dose calculation algorithms differ in their way of handling to this complex situation and thus should be always be evaluated. A clinically relevant example is the planning of a SBRT treatment for a small lung tumor, for which small margins and hypofractionated doses are frequently used. ${ }^{6}$

Figure (3) shows profile taken across the target calculated with clinical algorithms implemented in our planning system. Discrepancy between the differences algorithms in dose calculation within the target can be remarkably noticed as can demonstrated also in the histogram. The convolution algorithm showed overestimation of dose compared to superposition and fast superposition. These differences were not unexpected, but the magnitudes of the discrepancies are remarkable. ${ }^{1}$ The phantoms were deliberately designed to include a worst case scenario (beam traversing long low density path), resulting in a large discrepancy which was tabulated in table (1). It should be mentioned that this study did not consider complications resulting from tumor motion. We wanted to focus on the discrepancy resulting from the use of different algorithms.

The clinically relevant problem concerning the dose to the tumor is the under dosage that may arise when convolution is used, especially when the lung tumor is deeply seated within the lung, thus beam is traversing a long distance within low density path, which can have a large negative effect on tumor local control probability. ${ }^{1}$

\section{CONCLUSION}

There is a significant dose overestimation by Convolution in the heterogeneous geometries, especially in low-density; the discrepancy will be magnified as the beam traverses longer distance within low density tissues. The measurement showed that the superposition was the most accurate algorism in case of low density heterogeneity situation.

\section{REFERENCES}

1 L.R. Aarup, A.E. Nahum, C. Zacharatou, T. Juhler-Nottrup, T. Knoos, H. Nystrom, L. Specht, E. Wieslander, S.S. Korreman, "The effect of different lung densities on the accuracy of various radiotherapy dose calculation methods: implications for tumour coverage," Radiotherapy and oncology : journal of the European Society for Therapeutic Radiology and Oncology 91, 405-414 (2009).

2 B. Vanderstraeten, N. Reynaert, L. Paelinck, I. Madani, C. De Wagter, W. Gersem, W. De Neve, H. Thierens, "Accuracy of patient dose calculation for lung IMRT: A comparison of Monte Carlo, convolution/superposition, and pencil beam computations," Medical physics 33, 3149-3158 (2006).

3 J. Van Dyk, The modern technology of radiation oncology : a compendium for medical physicists and radiation oncologists. (Medical Physics Pub., Madison, Wis., 1999).

4 P.J. Levitt SH, Perez CA, Vijayakumar S. , "Technical basics of radiation therapy practical clinical application.," 135-136 (2006).

5 B. Fraass, K. Doppke, M. Hunt, G. Kutcher, G. Starkschall, R. Stern, J. Van Dyke, "American Association of Physicists in Medicine radiation therapy committee task group 53: Quality assurance for clinical radiotherapy treatment planning," Medical physics 25, 1773-1829 (1998).

6 G.X. Ding, D.M. Duggan, B. Lu, D.E. Hallahan, A. Cmelak, A. Malcolm, J. Newton, M. Deeley, C.W. Coffey, "Impact of inhomogeneity corrections on dose coverage in the treatment of lung cancer using stereotactic body radiation therapy," Medical physics 34, $2985-$ 2994 (2007).

7 U. Haedinger, T. Krieger, M. Flentje, J. Wulf, "Influence of calculation model on dose distribution in stereotactic radiotherapy for pulmonary targets," International journal of radiation oncology, biology, physics 61, 239-249 (2005).

8 A.T. Hansen, J.B. Petersen, M. Hoyer, J.J. Christensen, "Comparison of two dose calculation methods applied to extracranial stereotactic radiotherapy treatment planning," Radiotherapy and Oncology 77, 96-98 (2005).

9 I. Lax, V. Panettieri, B. Wennberg, M. Amor Duch, I. Naslund, P. Baumann, G. Gagliardi, "Dose distributions in SBRT of lung tumors: Comparison between two different treatment planning algorithms and Monte-Carlo simulation including breathing motions," Acta oncologica 45, 978-988 (2006).

10 V. Panettieri, B. Wennberg, G. Gagliardi, M.A. Duch, M. Ginjaume, I. Lax, "SBRT of lung tumours: Monte Carlo simulation with PENELOPE of dose distribu- 
tions including respiratory motion and comparison with different treatment planning systems," Physics in medicine and biology 52, 4265-4281 (2007).

11 P.B. Mark Buyyounouski, et al. , "Stereotactic Body Radiotherapy (SBRT) For Lung Cancer," Report of the ASTRO Emerging Technology Committee (ETC).

12 K.R. Muralidhar, N.P. Murthy, A.K. Raju, N. Sresty, "Comparative study of convolution, superposition, and fast superposition algorithms in conventional radiotherapy, three-dimensional conformal radiotherapy, and intensity modulated radiotherapy techniques for various sites, done on CMS XIO planning system," Journal of medical physics / Association of Medical Physicists of India 34, 12-22 (2009)

13 F.M. Khan, The physics of radiation therapy, 3rd ed. (Lippincott Williams \& Wilkins, Philadelphia, 2003).

14 M.M. Miften, A.W. Beavis, L.B. Marks, "Influence of dose calculation model on treatment plan evaluation in conformal radiotherapy: a three-case study," Medical dosimetry : official journal of the American Association of Medical Dosimetrists 27, 51-57 (2002).

15 A. Ahnesjo, "Collapsed cone convolution of radiant energy for photon dose calculation in heterogeneous media," Medical physics 16, 577-592 (1989).

16 E. Heath, J. Seuntjens, "A direct voxel tracking method for four-dimensional Monte Carlo dose calculations in deforming anatomy," Medical physics 33, 434-445 (2006).

17 N. Hodapp, “[The ICRU Report 83: prescribing, recording and reporting photon-beam intensity-modulated radiation therapy (IMRT)]," Strahlenther Onkol 188, 97-99 (2012).

18 M. Yoon, S.Y. Park, D. Shin, S.B. Lee, H.R. Pyo, D.Y. Kim, K.H. Cho, "A new homogeneity index based on statistical analysis of the dose-volume histogram," Journal of applied clinical medical physics / American College of Medical Physics 8, 9-17 (2007). 